\section{Zu Wirksamkeit und Folgen von preispolitischen Maßnah- men im Verkehrsbereich}

\author{
Von Hartmut Kuhfeld, Holger Schlör und \\ Ulrich Voigt, Deutsches Institut für Wirt- \\ schaftsforschung
}

Das Bundesministerium für Verkehr, Bauund Wohnungswesen hat kürzlich im „Verkehrsbericht 2000" Szenarien und Prognosen zur Verkehrsentwicklung bis zum Jahr 2015 vorgelegt. Danach ist - vor allem im Güterverkehr - noch mit einer kräftigen Zunahme der Leistungen im Straßenverkehr zu rechnen. Die weiter steigenden Belastungen des Straßennetzes werden voraussichtlich allerdings nicht durch entsprechenden Ausbau der Kapazitäten allein bewältigt werden können. In diesem Zusammenhang werden seit geraumer Zeit Maßnahmen und Modelle zur Bewirtschaftung der Verkehrsinfrastruktur diskutiert. Auch zur Verbesserung der ökologischen Verträglichkeit des Verkehrs werden preispolitische Maßnahmen untersucht. Im folgenden Beitrag werden dazu Ergebnisse einer Studie des DIW präsentiert.

\section{Ziele und Instrumente von preispolitischen Maßnahmen im Verkehrssektor}

Preispolitische Maßnahmen haben in der aktuellen verkehrspolitischen Diskussion einen erheblichen Stellenwert. Die im Gesetz zur ökologischen Steuerreform enthaltenen Erhöhungen der Mineralölsteuer sowie die Empfehlungen der Regierungskommission Verkehrsinfrastrukturfinanzierung, vom Jahre 2003 an eine streckenbezogene Benutzungsgebühr für schwere Lkw (ab $12 \mathrm{t}$ zGG) auf Bundesautobahnen in Höhe von 25 Pfennigen je Fahrzeugkilometer einzuführen, gewinnen vor dem Hintergrund der Mineralölverteuerungen im laufenden Jahr zusätzliche Aufmerksamkeit und finanzielle Bedeutung. Dabei steht neben den verkehrlichen Wirkungen vor allem auch die Frage der ökonomischen Folgen im Vordergrund des Interesses.

Das DIW hat vor einiger Zeit im Rahmen des Projektes „Ökonomische Folgenanalyse zur
Entlastung des Verkehrsnetzes und zur Verlagerung von Straßenverkehr auf umweltfreundlichere Verkehrsträger" des Büros für Technikfolgen-Abschätzung beim Deutschen Bundestag (TAB) eine verkehrliche und ökonomische Folgenanalyse von verkehrsbezogenen Preisstrategien erarbeitet. ${ }^{1}$

Dabei ging es um Maßnahmen

- zur zeitlichen Steuerung der Straßenbelastung (verschiedene Formen des Road Pricing) und

- $\quad$ zur Vermeidung oder zur Verlagerung von Straßenverkehr auf andere Verkehrsträger (etwa durch Steuern und Gebühren).

Basisjahr für die quantitativen Untersuchungen war in dieser Studie das Jahr 1994. Seitdem hat sich - vor allem im Güterverkehr - die Problemsituation durch Deregulierung und starke Zunahme des Straßenverkehrs weiter verschärft. Die in der Studie behandelten Maßnahmen und deren zu Grunde gelegte Intensität lassen die Ergebnisse jedoch auch aus heutiger Sicht als relevant erscheinen. Die analytischen Zusammenhänge dürften nach wie vor Gültigkeit haben.

Grundsätzlich sollten Strategien zur Gestaltung des Verkehrssystems integriert für alle Bereiche der Verkehrspolitik (Investitionspolitik, Preispolitik, Ordnungspolitik, organisatorische Maßnahmen, Öffentlichkeitsarbeit) und verkehrsträgerübergreifend erarbeitet werden. Es wurde daher davon ausgegangen, dass eine Entlastung des Verkehrsnetzes und die Verlagerung von Straßenverkehr auf umweltfreundlichere Verkehrsträger nicht allein durch eine monetäre Steuerung erreicht werden soll. Vielmehr werden die Auswirkungen einer Verteuerung von Verkehrsleistungen in der Studie für das Büro für Technikfolgen-Abschätzung beim Deutschen Bundestag (TAB) innerhalb eines konsistenten Maßnahmenbündels aus verschiedenen Politikbereichen untersucht. Die Einbettung der Preispolitik in einen abgestimmten „Instrumenten-Mix“ gewährleistet, dass die Erreichung der angestrebten Ziele nicht durch gegenläufige Wirkungen behindert wird, sondern sich die Wirkungen einzelner Maßnahmen ergänzen und gegenseitig verstärken. Diese Synergiewirkungen wiederum gestatten es, Preiserhöhungen vergleichsweise gering zu halten und damit den 
betroffenen Wirtschaftssubjekten (Haushalte und Unternehmen) unnötige Schocks zu ersparen.

Für preispolitische Instrumente zur Entlastung des Straßennetzes und zur Beeinflussung der Verkehrsteilung gibt es eine Reihe von Ansatzpunkten: Die Maßnahmen können sich auf den Erwerb und das Halten von Kraftfahrzeugen beziehen, sie können am Kraftstoffverbrauch ansetzen oder an der zurückgelegten Strecke. Schließlich kann auch das Befahren bestimmter Gebiete sowie das Abstellen und Parken der Fahrzeuge bewirtschaftet werden.

Hinsichtlich der Art der preispolitischen Maßnahmen sind Steuern, Abgaben und Gebühren, monetäre Kompensationen (personenbezogene Transfers, Subventionen, Steuerbegünstigungen) sowie als Mengensteuerung mit Preiswirkungen die Vergabe von Lizenzen zu unterscheiden.

Die verschiedenen Maßnahmen wirken jeweils in unterschiedlicher Weise auf die Verkehrsnachfrage, so dass ein Instrumentenmix generell eine breitere Wirkung verspricht als die Einführung oder Verstärkung einer einzelnen preispolitischen Regelung.

Im Einzelnen wurden in der Studie zunächst folgende preispolitische Instrumente im Hinblick auf ihre Wirkungsweise und ihre Bedeutung diskutiert:

- Kraftfahrzeugsteuer,

- Mineralölsteuer,

- Kilometerpauschale,

- Nahverkehrsabgabe,

- Straßenbenutzungsgebühren (Road Pricing),

- Parkraumbewirtschaftung,

- Schwerverkehrsabgabe,

- Lizenzen,

- $\quad$ sonstige Regelungen.

\section{Verkehrspolitische Szenarien}

Für die Bildung von verkehrspolitischen Szenarien wurden einzelne Maßnahmen gebündelt. So wurden verschiedene Formen des Road Pricing (vorwiegend mit Hilfe moderner IuK-Techniken) als wesentliche Instrumente zur Verkehrslenkung einbezogen. Die Straßenbenutzungsgebühren wurden in unterschiedlichem Umfang mit einer Erhöhung der Mineralölsteuer verknüpft, um Anreize zur Minderung des Kraftstoffverbrauchs in die Szenarien aufzunehmen. Als ergänzende Maßnahme wurde in einem Szenario auch eine verstärkte, restriktive Parkraumbewirtschaftung zugrunde gelegt. Die z. T. ebenfalls kostenwirksamen flankierend notwendigen Maßnahmen (z. B. Ausbau des Öffentlichen Verkehrs) und die möglichen telematischen Zusatzdienste (z. B. automatische Stauwarninformationen und Routenempfehlungen) ergeben sich aus der Grundannahme der gesamten TAB-Untersuchung, die Preispolitik innerhalb eines abgestimmten Maßnahmenbündels $\mathrm{zu}$ betrachten sowie aus den Ergebnissen zur Abschätzung der verkehrlichen Wirksamkeit.

Beim Road Pricing auf der Grundlage von modernen IuK-Techniken wird in den Szenarien unterschieden zwischen der Einführung solcher Systeme auf den Bundesautobahnen einerseits und in Ballungsgebieten andererseits. Dabei werden unterschiedliche Preisbildungsverfahren verwendet. Während auf den Autobahnen die Benutzergebühren entfernungsabhängig gestaltet werden, wird für Ballungsgebiete das so genannte Cordon Pricing, d. h. eine Preiserhebung für das Einfahren in bestimmte Gebiete zugrunde gelegt.

Für die Strategiebeschreibung erscheinen drei Szenarien einerseits als ausreichend, um genügend Differenzierungen für unterschiedliche Maßnahmenausprägungen zu ermöglichen. Andererseits bleibt die Analyse damit in einem überschaubaren Rahmen und führt zu Ergebnissen, die als deutliche Alternativen voneinander abgesetzt sind.

Hinsichtlich der Höhe der Abgaben und Steuerbelastungen wurden für die einzelnen Szenarien Größenordnungen angenommen, die sich sowohl von der Situation im Basisjahr 1994 als auch untereinander deutlich unterscheiden. Dabei führt die Kombination einer Verdoppelung bzw. Vervierfachung der Mineralölsteuer mit weiteren preispolitischen Maßnahmen zu einer deutlich höheren finanziellen Belastung des Kraftfahrzeugverkehrs als bisher. Mit diesen Annahmen wird gewährleistet, dass die Folgeanalysen nicht lediglich graduelle Fortschreibungen des Status-quo, sondern deutlich voneinander abgesetzte, unterschiedliche Politikkonzeptionen quantitativ bewerten.

Im ersten Szenario wird unterstellt: 
- Einführung von elektronischem Road Pricing auf Bundesautobahnen und ausgewählten Bundesfernstraßen (zur Verhinderung von Ausweichreaktionen) für $\mathrm{Pkw}$ in Höhe von durchschnittlich $20 \mathrm{Pf} / \mathrm{km}$ und für Lkw in Höhe von $80 \mathrm{Pf} / \mathrm{km}$;

- Einführung von Cordon Pricing in den Innenstadtbereichen der Ballungsgebiete in Höhe von durchschnittlich $5 \mathrm{DM}$ je $\mathrm{Zu}-$ fahrt in die Innenstadt,

- Mineralölsteuererhöhung auf 2 DM je Liter Vergaserkraftstoff und (energieäquivalent) auf 2,18 DM je Liter Diesel.

Im zweiten Szenario wird angenommen, dass die Einführung von Straßenbenutzungsgebühren für Pkw vorerst nicht erfolgt. Stattdessen wird für Pkw eine Autobahn-Vignette vorgesehen. Die wesentlichen preisrelevanten Annahmen sind damit:

- Einführung von elektronischem Road Pricing auf Bundesautobahnen und ausgewählten Bundesfernstraßen (zur Verhinderung von Ausweichreaktionen) für Lkw in Höhe von $80 \mathrm{Pf} / \mathrm{km}$, Autobahn-
Vignette für Pkw zum Preis von $300 \mathrm{DM} / \mathrm{Jahr}$;

- Einführung von Cordon Pricing in Ballungsgebieten in Höhe von durchschnittlich 5 DM je Zufahrt in die Innenstadt;

- Mineralölsteuererhöhung auf $2 \mathrm{DM}$ je Liter Vergaserkraftstoff und (energieäquivalent) auf 2,18 DM je Liter Diesel.

Im dritten Szenario wird untersucht, inwieweit die beabsichtigte Verkehrsbeeinflussung ohne Investitionen in eine Road PricingInfrastruktur - allein durch eine drastische Erhöhung der Mineralölsteuer erreichbar ist. Cordon Pricing als Steuerungsinstrument der Zufahrt in die Innenstädte soll durch eine verstärkte Parkraumbewirtschaftung und Zufahrtsbeschränkungen ersetzt werden. Vorgesehen wird

- verstärkte Parkraumbewirtschaftung in Ballungsgebieten (4 DM/Stunde);

- Mineralölsteuererhöhung auf 4 DM je Liter Vergaserkraftstoff und (energieäquivalent) auf 4,36 DM je Liter Diesel (vgl. Tab. 1).

Tab. 1: Preispolitische Annahmen zu den Szenarien

\begin{tabular}{|c|c|c|c|c|}
\hline \multirow{2}{*}{$\begin{array}{l}\text { Mineralölsteuer DM/I } \\
\end{array}$} & \multirow{3}{*}{$\begin{array}{c}\text { Ist } 1994 \\
0,98\end{array}$} & \multicolumn{3}{|c|}{$\begin{array}{c}\text { Maßnahmen zu Szenarien } \\
1 \quad 2 \\
\begin{array}{c}\text { nach } 15 \text { Jahren } \\
\text { M }\end{array}\end{array}$} \\
\hline & & & & \\
\hline Vergaserkraftstoff & & 2,00 & 2,00 & 4,00 \\
\hline Dieselkraftstoff & 0,62 & 2,18 & 2,18 & 4,36 \\
\hline \multicolumn{5}{|l|}{ Tankstellenpreis } \\
\hline Vergaserkraftstoff & 1,55 & 2,72 & 2,72 & 5,01 \\
\hline Dieselkraftstoff & 1,15 & 2,94 & 2,94 & 5,45 \\
\hline \multicolumn{5}{|l|}{ Autobahnbenutzung } \\
\hline $\mathrm{Pkw} \quad \mathrm{DM} / \mathrm{km}$ & - & 0,20 & - & - \\
\hline Lkw $\mathrm{DM} / \mathrm{km}$ & - & 0,80 & 0,80 & - \\
\hline \multicolumn{5}{|l|}{ Vignette DM/Jahr } \\
\hline Lkw & 2200 & 5000 & 5000 & 3000 \\
\hline Pkw & & & 300 & \\
\hline Parkraumbewirtschaftung DM/Std. & & & & 4,00 \\
\hline Ballungsraumzufahrt DM & - & 5,00 & 5,00 & - \\
\hline
\end{tabular}




\section{Akzeptanz der Maßnahmen}

Mit den kräftigen Belastungserhöhungen, die in den Szenarien zugrunde gelegt werden, wird natürlich auch die Frage nach der verkehrspolitischen Bedeutung und der Akzeptanz der Maßnahmen aufgeworfen.

Generell ist darauf hinzuweisen, dass die getroffenen Annahmen zunächst als Grundlage für die Schätzung von Reaktionen der betroffenen Wirtschaftssubjekte (Haushalte und Unternehmen) dienen und nicht den Charakter von politischen Handlungsempfehlungen haben. Ziel der Untersuchung ist es, die betroffenen Gruppen einzugrenzen und den Grad der Betroffenheit sowie die Reaktionsmöglichkeiten festzustellen. Eine solche Bewertung von unterschiedlichen politischen Handlungskonzepten lässt den Einfluss der untersuchten Parameter unterscheidbarer darstellen, wenn deutliche Preissignale vorgegeben werden, als bei einer Betrachtung von nur graduellen Änderungen. Erst wenn die Ergebnisse der Bewertung vorliegen, können in einer zweiten Stufe die Zumutbarkeit und die Akzeptanz diskutiert werden.

Über diese generellen Aspekte hinaus ist bei der Betrachtung zu berücksichtigen, dass

- die Bedeutung der fahrtenbezogenen Kosten sich in einer Gesamtkostenbetrachtung unter Einschluss der anderen Kostenarten (wie Abschreibungen, Reparaturen, Versicherungen, Personalkosten) relativiert,

- nach bisherigen Untersuchungen nur durch deutliche Preissignale Verhaltensänderungen erreichbar sind,

- für die Implementation der Maßnahmen ein stufenweises Vorgehen in einem Zeitraum von etwa 15 Jahren zu Grunde gelegt wird, so dass abrupte Schocks weitgehend vermieden werden und die betroffenen Wirtschaftssubjekte im Zeitablauf mit technischen und verhaltensbezogenen Anpassungen an die veränderte Kostensituation reagieren können sowie

- im Anpassungszeitraum durch Einkommenssteigerungen Spielräume geschaffen werden, mit denen die durch die preispolitischen Maßnahmen induzierten Strukturveränderungen ,,abgefedert“ werden können.
Mit den zusätzlichen Steuern und Abgaben sollen per saldo keine zusätzlichen Belastungen von Unternehmen und Haushalten verbunden sein. Daher sind entsprechende Kompensationen vorzusehen. Diese können in indirekter Weise, z. B. als Förderungsmaßnahmen für den öffentlichen Verkehr oder in bestimmten Formen der direkten monetären Kompensation erbracht werden. Hierzu wurden in der vorliegenden Studie keine Festlegungen getroffen.

\section{Methodische Vorgehensweise}

Die Maßnahmen wurden im Rahmen von verkehrlichen und ökonomischen Wirkungsanalysen untersucht. Dabei bestehen zwischen beiden Teilen enge Interdependenzen, da zum einen ökonomische Wirkungen verkehrsbezogene Verhaltensweisen stimulieren und andererseits verkehrliche Reaktionen ökonomische Wirkungen (z. B. auf Ausgaben oder Investitionen) haben.

Ein anderer Aspekt ist die Fristigkeit der Maßnahmenwirkungen. Diese ergeben sich in der Realität erst im Laufe eines längeren Anpassungsprozesses von etwa 10 bis 15 Jahren. Bei der Analyse der verkehrlichen Wirksamkeit wird hier davon ausgegangen, dass die Reaktionen auf die veränderten Preise bereits abgeschlossen sind. Es wird also nicht der zeitliche Verlauf der Veränderungen beschrieben, sondern das Ergebnis nach Ablauf aller Anpassungsprozesse. Die Maßnahmen werden dabei unter den im Basisjahr vorliegenden Rahmenbedingungen analysiert. Dies bedeutet, dass die verkehrlichen und ökonomischen Wirkungen auf Ausgangsdaten für das Jahr 1994 bezogen werden. Damit wird der Tatsache Rechnung getragen, dass entsprechende Prognoseergebnisse nur in aufwendigen Studien zu gewinnen wären und in der hier verwendeten Detaillierung - falls überhaupt prognostizierbar - kaum belastbar wären. Diese Vorgehensweise geht von der Annahme aus, dass unter ex-post Bedingungen wirksame Maßnahmen dies auch in Zukunft sein werden. 


\section{Verkehrliche und ökonomische Wirkungen der Szenarien im Personenverkehr ...}

Tabelle 2 enthält die zusammengefassten Ergebnisse der Szenarien für Verkehrsausgaben, Verkehrsleistungen, Fahrleistungen und Kraftstoffverbrauch im Personenverkehr. Diese zeigen, dass die Wirkungen zwischen den Szenarien zum Teil erheblich differieren.

Während die Ausgaben der Verkehrsteilnehmer je nach Szenario gegenüber 1994 um $10 \%$ bis $16 \%$ und damit um eine ähnliche Größenordnung zunehmen, unterscheiden sich die verkehrlichen Wirkungen insbesondere zwischen den Szenarien 1 und 2 einerseits und Szenario 3 andererseits vor allem hinsichtlich der Veränderungen der gesamten Pkw-Fahrleistung deutlich. Im Szenario 3 bewirkt vor allem die kräftige Mineralölsteuererhöhung eine Reduktion der Fahrleistungen um mehr als ein Fünftel; die anderen beiden Szenarien, in denen wesentliche Instrumente eine moderatere Erhöhung der Mineralölsteuer und die Bewirtschaftung des Autobahnnetzes sind, führen dagegen zu einer deutlich geringeren Verminderung, und zwar um $10 \%$ im Szenario 1 und um $8 \%$ im Szenario 2 .

Bei den Verkehrsleistungen (Personenkilometer) sind die Rückgänge im motorisierten Individualverkehr durchweg weniger stark ausgeprägt, weil ein Teil der vermiedenen Fahrleistung durch eine verbesserte Auslastung der Fahrzeuge kompensiert wird. Hier ergibt sich also eine gewisse Entkoppelung von Verkehrs- und Fahrleistungen. Die Zunahme der Leistungen bei den öffentlichen Verkehrsmitteln aufgrund von Verlagerungen des PkwVerkehrs beträgt im Szenario 3 etwa ein Drittel, in den anderen Szenarien deutlich weniger (Szenario 1: $16 \%$, Szenario 2: $11 \%$ ). Diese Unterschiede korrespondieren in etwa mit den Veränderungen bei den Pkw-Verkehrsleistungen.

Bemerkenswerte Ergebnisse ergeben sich bei den Minderungswirkungen für den Kraftstoffverbrauch von Pkw. Die Kombination der technischen Maßnahmen zur Verbesserung des spezifischen Energieverbrauchs mit der Fahrleistungsreduktion führt im Szenario $3 \mathrm{zu}$ einem Rückgang um $57 \%$. In den anderen beiden Szenarien beträgt die Verminderung jeweils mehr als ein Drittel (37\% bzw. $36 \%$ ).
Diese zum Teil außerordentlich starke Reduktion des Kraftstoffverbrauchs wird in den hier betrachteten Szenarien durch die Verteuerung des Pkw-Verkehrs induziert, deren Ausmaß nicht allein an den Steigerungen der entsprechenden Ausgaben abzulesen ist. Berücksichtigt man neben der Ausgabenerhöhung auch die Verminderung der Fahrleistungen, so ergeben sich Steigerungen der Kosten je Pkw-Kilometer gegenüber dem Wert von 1994

- $\quad$ im Szenario 1 von $24 \%$,

- $\quad$ im Szenario 2 von $19 \%$,

- $\quad$ im Szenario 3 von $48 \%$.

Neben den globalen, auf das gesamte Verkehrssystem in der Volkswirtschaft bezogenen Maßnahmen sind in den Szenarien auch einige lokal wirkende Maßnahmen (Road Pricing und Parkraumbewirtschaftung in den Ballungsgebieten) enthalten, die in Bezug auf die Gesamtfahrleistungen nur verhältnismäßig geringe Effekte haben, für den Verkehr in den betroffenen Städten aber von erheblicher Bedeutung sind.

Beide Maßnahmen erbringen in der hier zugrunde gelegten Ausgestaltung eine weitgehend gleiche Fahrleistungsreduktion von rund 3 Mrd. Kilometern. Ihre Wirkungen für die jeweilige örtliche Verkehrssituation sind wegen unterschiedlicher räumlicher Abgrenzungen jedoch nur schwer miteinander zu vergleichen. Während der wichtigste Effekt des innerstädtischen Road Pricing in der zeitlichen Verlagerung von Fahrten und der Glättung von Verkehrsspitzen besteht, dürfte demgegenüber die Parkraumbewirtschaftung stärker die Entscheidung über das Ziel einer Fahrt und das benutzte Verkehrsmittel beeinflussen. Letztlich werden die Effekte jedoch maßgeblich von der tariflichen Ausgestaltung der Systeme abhängen und sind von den jeweiligen örtlichen Gegebenheiten mit abhängig. 
Tab. 2: Verkehrsausgaben, Verkehrsleistungen, Fahrleistungen und Kraftstoffverbrauch im Jahre 1994 in Deutschland und in den Szenarien

\begin{tabular}{|c|c|c|c|c|c|}
\hline & Einheit & 1994 & Szenario 1 & Szenario 2 & Szenario 3 \\
\hline \multicolumn{6}{|l|}{ Verkehrsausgaben } \\
\hline Motorisierter Individualverkehr 1) & Mrd. DM & 295,4 & 332,2 & 325,0 & 341,7 \\
\hline Eisenbahn und ÖSPV 2) & Mrd. DM & 25,2 & 29,0 & 28,1 & 33,5 \\
\hline Verkehr insgesamt & Mrd. DM & 320,6 & 361,1 & 353,1 & 375,2 \\
\hline \multicolumn{6}{|l|}{ Verkehrsleistungen } \\
\hline Motorisierter Individualverkehr 1) & Mrd. Pkm & 749,2 & 697,2 & 709,4 & 631,9 \\
\hline Eisenbahn und ÖSPV 2) & Mrd. Pkm & 140,3 & 162,9 & 156,3 & 185,9 \\
\hline Fußgänger, Fahrrad-Verkehr 3) & Mrd. Pkm & 54,5 & 60,3 & 60,3 & 71,2 \\
\hline Verkehr insgesamt & Mrd. Pkm & 944,0 & 920,3 & 926,1 & 889,1 \\
\hline \multicolumn{6}{|l|}{ Fahrleistungen } \\
\hline Motorisierter Individualverkehr 1) & Mrd. km & 505,7 & 456,1 & 467,4 & 394,5 \\
\hline $\begin{array}{c}\text { dav Bundesautobahnen } \\
\text { Übrige Straßen }\end{array}$ & $\begin{array}{l}\text { Mrd. km } \\
\text { Mrd. km }\end{array}$ & $\begin{array}{l}145,2 \\
360,5\end{array}$ & $\begin{array}{l}123,8 \\
332,3\end{array}$ & $\begin{array}{l}135,1 \\
332,3\end{array}$ & $\begin{array}{l}113,7 \\
280,8\end{array}$ \\
\hline \multicolumn{6}{|l|}{ Kraftstoffiverbrauch } \\
\hline Motorisierter Individualverkehr 1) & Mill. I & 38.293 & 24.063 & 24.684 & 16.306 \\
\hline & Verkehrsausgaben & & eränderung & gegenüber 1 & $1994-$ \\
\hline Motorisierter Individualverkehr 1) & $\%$ & $x$ & 12,4 & 10,0 & 15,7 \\
\hline Eisenbahn und ÖSPV 2) & $\%$ & $x$ & 14,9 & 11,5 & 32,7 \\
\hline Verkehr insgesamt & $\%$ & $x$ & 12,6 & 10,1 & 17,0 \\
\hline \multicolumn{6}{|l|}{ Verkehrsleistungen } \\
\hline Motorisierter Individualverkehr 1) & $\%$ & $x$ & $-6,9$ & $-5,3$ & $-15,7$ \\
\hline Eisenbahn und ÖSPV 2) & $\%$ & $x$ & 16,1 & 11,4 & 32,5 \\
\hline Fußgänger, Fahrrad-Verkehr 3) & $\%$ & $x$ & 10,6 & 10,6 & 30,6 \\
\hline Verkehr insgesamt & $\%$ & $x$ & $-2,5$ & $-1,9$ & $-5,8$ \\
\hline \multicolumn{6}{|l|}{ Fahrieistungen } \\
\hline Motorisierter Individualverkehr 1) & $\%$ & $x$ & $-9,8$ & $-7,6$ & $-22,0$ \\
\hline $\begin{array}{c}\text { dav Bundesautobahnen } \\
\text { Übrige Straßen }\end{array}$ & $\begin{array}{l}\% \\
\%\end{array}$ & $\begin{array}{l}x \\
x\end{array}$ & $\begin{array}{r}-14,8 \\
-7,8\end{array}$ & $\begin{array}{l}-7,0 \\
-7,8\end{array}$ & $\begin{array}{l}-21,7 \\
-22,1\end{array}$ \\
\hline Kraftstoffverbrauch & & & $\cdot$ & & \\
\hline Motorisierter Individualverkehr 1) & $\%$ & $x$ & $-37,2$ & $-35,5$ & $-57,4$ \\
\hline
\end{tabular}


Für die Bewertung der ökonomischen Effekte werden die mit den preispolitischen Maßnahmen verbundenen zusätzlichen Ausgaben der privaten Haushalte einerseits sowie der Unternehmen und Selbständigen andererseits quantifiziert und zu den aktuellen Ausgaben in Beziehung gesetzt. Bei den Unternehmen sind Pkw-Fahrten in der Regel Vorleistungen im Rahmen der betrieblichen Leistungserstellung. Die Ergebnisse zeigen, dass die Belastungen durch die Verteuerungsmaßnahmen nur eine geringe Bedeutung innerhalb des Produktionsprozesses haben (bis zu 0,3\% des Produktionswertes). Infolgedessen dürften von den Maßnahmen kaum gravierende Auswirkungen auf die Produktion ausgehen.

Bei den privaten Haushalten liegen die gesamten Ausgaben für Pkw-Haltung und Nutzung nach den verschiedenen Anpassungsreaktionen in den drei Szenarien kaum noch auseinander. Gegenüber dem Ausgangswert im Jahre 1994 beträgt die Erhöhung

$\begin{array}{lll}\text { - } & \text { im Szenario 1 } & 10 \%, \\ \text { - } & \text { im Szenario 2 } & 8 \% \text { und } \\ \text { - } & \text { im Szenario 3 } & 11 \% .\end{array}$

Es wird deutlich, dass die privaten Haushalte im Rahmen ihrer gesamten Budgetentscheidungen die Ausgaben für Pkw-Mobilität nur begrenzt erhöhen können. Stärkere Verteuerungen bewirken stärkere Effizienz-, Verlagerungs- und Vermeidungsreaktionen, um die monetären Belastungen zu vermindern.

Die Ausgaben für die gesamten Verkehrsleistungen steigen für die Szenarien 1 und $2 \mathrm{im}$ gleichen Umfang wie die Ausgaben für PkwHaltung und -Nutzung (10\% bzw. $8 \%)$. Im Szenario 3 liegt die Zunahme leicht darüber, nämlich bei $12 \%$. Damit ergeben sich Größenordnungen für die Belastung der Haushaltsbudgets, die im Mittel als realisierbar erscheinen. Bezogen auf die gesamten Ausgaben der privaten Haushalte, entfallen auf die Verkehrsausgaben Anteile von 15,5\% (Szenario 2), 15,8\% (Szenario 1) und 16,1\% (Szenario 3). Im Vergleich zum Ausgangswert von 1994 (14,3\%) erscheinen die durch die Verteuerungsmaßnahmen induzierten Veränderungen als moderat und bleiben im Rahmen der in der Vergangenheit beobachteten Veränderungen. So ist der Anteil der Verkehrsausgaben am Konsum der privaten Haushalte von 1980 bis 1990 um 2,2\% gestie- gen. Im Jahr 1990 lag er mit 15,0 \% bereits nahe bei den hier ermittelten Werten für die Szenarien.

Um die Bedeutung der zusätzlichen PkwAusgaben für Haushalte mit unterschiedlicher Einkommenssituation zu kennzeichnen, wurden entsprechende haushaltsbezogene Daten aus der Einkommens- und Verbrauchsstichprobe 1988 (für die alten Bundesländer) ausgewertet. Als Indikator für die finanzielle Belastung der Haushalte durch die von den preispolitischen Maßnahmen verursachten zusätzlichen Kosten wurde das Verhältnis der szenariobedingten zusätzlichen Pkw-Ausgaben und der jeweiligen Haushaltsersparnis gebildet. Die Ersparnis ist der einzige kurzfristig variable Parameter des Haushaltsbudgets und eignet sich daher als Bezugsgröße zur Kennzeichnung des Anpassungsund Veränderungsdrucks, der von der Kostenerhöhung ausgeht und längerfristig zu Veränderungen bzw. Umschichtungen in den Haushaltsbudgets führt.

Anhand dieser Relation wurden die Haushalte in 4 Belastungsgruppen eingeteilt, für die die Reaktionen jeweils gesondert betrachtet wurden:

- Gruppe 1 umfasst diejenigen Haushalte, bei denen die zusätzlichen monatlichen Kosten mehr als $80 \%$ der Ersparnis ausmachen. Hier wird davon ausgegangen, dass die durch die Szenario-Maßnahmen verursachten Kosten nicht durch Haushaltsbudgetumschichtungen ausgeglichen werden können. Die Haushalte dieser Gruppe werden überwiegend auf die Haltung und Nutzung eines eigenen Pkws verzichten.

- Gruppe 2 umfasst die Haushalte, deren zusätzliche Belastung zwischen 25 und $80 \%$ ihrer Ersparnis liegt.

- Gruppe 3 enthält die Haushalte mit verkehrlichen Mehrausgaben von 10 bis $25 \%$ der monatlichen Ersparnis.

- Gruppe 4 besteht aus den Haushalten, deren szenariobedingte Mehrausgaben bis zu $10 \%$ ihrer Ersparnis ausmachen.

Tabelle 3 zeigt die Verteilung der Haushaltseinkommensklassen auf die vier Gruppen. Die Zuordnung variiert zwischen den Szenarien etwas, da die preispolitischen Maßnahmen je 
nach ihrer Zusammensetzung zu unterschiedlichen Belastungserhöhungen führen:

- Auf die Gruppe 1 entfallen in allen Szenarien 1,9 Mill. Haushalte mit insgesamt 4,7 Mill. Haushaltsangehörigen. Dies entspricht $10 \%$ aller motorisierten Haushalte. Die Szenario-Maßnahmen verursachen bei diesen Haushalten zusätzliche Kosten, die durch Umschichtungen des Budgets in der Regel nicht mehr ausgeglichen werden können, da die durch die Szenario-
Maßnahmen verursachten Belastungen die Ersparnis z. T. bei weitem übertreffen. Diese Gruppe kann sich zu einem großen Teil einen eigenen Pkw nicht mehr leisten und muss verstärkt öffentliche Verkehrsmittel oder das Fahrrad benutzen bzw. ihre Wege zu Fuß zurücklegen.

- Gruppe 2 umfasst in allen Szenarien die Einkommensklassen zwischen 1800 und 3000 DM. Darauf entfällt etwa ein Viertel aller motorisierten Haushalte (5 Mill.) mit 12,2 Mill. Haushaltsangehörigen. Die

Tab. 3: Belastung von Haushaltsgruppen in den Szenarien

\begin{tabular}{|c|c|c|c|c|}
\hline \multirow{3}{*}{$\begin{array}{l}\text { Veränderung der MIV- } \\
\text { Ausgaben } \\
\text { in \% der Ersparnis }\end{array}$} & \multicolumn{4}{|c|}{ - Szenario 1 - } \\
\hline & Einkommen & Personen & Haushalte & Anteil der \\
\hline & DM & Mill. & Mill. & in $\%$ \\
\hline über $80^{1)}$ & bis 1800 & 4,7 & 1,9 & 10 \\
\hline $25-80$ & $1800-3000$ & 12,2 & 5,1 & 26 \\
\hline $10-25$ & $3000-4500$ & 13,6 & 5,6 & 29 \\
\hline unter 10 & über 4500 & 16,5 & 6,8 & 35 \\
\hline Haushalte Insgesamt & & 47 & 19,3 & 100 \\
\hline \multirow{3}{*}{$\begin{array}{l}\text { Veränderungen der } \\
\text { MIV-Ausgaben in \% } \\
\text { der Ersparnis }\end{array}$} & \multicolumn{4}{|c|}{ - Szenario 2 - } \\
\hline & Einkommen & Personen & Haushalte & Anteil der \\
\hline & $\mathrm{DM}$ & Mill. & Mill. & in $\%$ \\
\hline über $80^{1)}$ & bis 1800 & 4,7 & 1,9 & 10 \\
\hline $25-80$ & $1800-3000$ & 12,2 & 5,0 & 26 \\
\hline $10-25$ & $3000-4000$ & 9,4 & 3,9 & 20 \\
\hline unter 10 & über 4000 & 20,7 & 8,5 & 44 \\
\hline Haushalte Insgesamt & & 47 & 19,3 & 100 \\
\hline \multirow{3}{*}{$\begin{array}{l}\text { Veränderung der MIV- } \\
\text { Ausgaben in \% der } \\
\text { Ersparnis }\end{array}$} & \multicolumn{4}{|c|}{ - Szenario 3 - } \\
\hline & Einkommen & Personen & Haushalte & Anteil der \\
\hline & DM & Mill. & Mill. & in $\%$ \\
\hline über $80^{1)}$ & bis 1800 & 4,7 & 1,9 & 10 \\
\hline $25-80$ & $1800-3000$ & 12,2 & 5,0 & 26 \\
\hline $10-25$ & $3000-4500$ & 13,6 & 5,6 & 29 \\
\hline unter 10 & über 4500 & 16,5 & 6,8 & 35 \\
\hline Haushalte Insgesamt & & 47 & 19,3 & 100 \\
\hline
\end{tabular}


Haushaltsgruppe kann zusätzliche Belastungen grundsätzlich mit eigenen Mitteln ausgleichen. Die Reaktionen auf die Verteuerungen werden allerdings relativ stark ausfallen.

- In Gruppe 3 variiert die obere Einkommensgrenze zwischen den Szenarien etwas. Im Szenario 2, demjenigen mit der geringsten Gesamtbelastung, liegt sie bei $4000 \mathrm{DM}$, in den Szenarien 1 und 2 bei 4500 DM. Demzufolge entfallen auf diese Gruppe im Szenario 2 3,9 Mill. der motorisierten Haushalte $(20 \%)$ und den Szenarien 1 und 3 jeweils 5,6 Mill. (29\%). Bei den Angehörigen dieser Gruppe sind die Veränderungen bei den Verkehrsausgaben zwar spürbar, aber nicht einschneidend. Die Reaktionen dürften daher von mittlerer Stärke sein und sich möglicherweise eher auf den Freizeitverkehr als auf die ,notwendigen" Fahrten im Berufs- und im Ausbildungsverkehr beziehen.

- Gruppe 4 umfasst diejenigen Haushalte, deren Einkommen über den oberen Grenzen von Gruppe 3 liegt. Im Szenario 2 sind dies 8,5 Mill. motorisierte Haushalte (44\%), in den Szenarien 1 und 3 6,8 Mill. (35\%) mit 20,7 Mill. bzw. 16,5 Mill. Angehörigen. Die zusätzlichen Belastungen reduzieren hier die durchschnittliche monatliche Ersparnis nur um 5\%. Die PkwMobilität der Haushalte dürfte von den Maßnahmen nur geringfügig betroffen werden.

Zusammengefasst zeigen die Ergebnisse für die Haushaltskategorien, dass etwa 2 Mill. motorisierte Haushalte $(10 \%$ der motorisierten Haushalte) mit insgesamt knapp 5 Mill. Haushaltsangehörigen die Belastungen kaum mit eigenen Mitteln ausgleichen können und daher zu einem großen Teil gezwungen sein dürften, auf den Pkw zu verzichten. Unter den sozialen Gruppen sind die Arbeitslosen am einschneidendsten von den szenariobedingten Mehrausgaben betroffen; $70 \%$ aller motorisierten Haushalte dieser Gruppe fallen in die Belastungskategorie, in der das eigene Fahrzeug nur schwierig zu halten sein dürfte, obwohl gerade für die Rückkehr in den Produktionsprozess räumliche Mobilität von besonderer Bedeutung ist.
Während also im gesamtwirtschaftlichen Mittel die von den Verteuerungsmaßnahmen ausgehenden Impulse nach einem längerfristigen Anpassungsprozess keine gravierenden Auswirkungen auf die Konsumstruktur haben würden, macht die Betrachtung der Haushaltsgruppen deutlich, dass sich hinter den Durchschnittswerten unterschiedliche Entwicklungen in einzelnen Bevölkerungskategorien verbergen. Ein relevanter Teil der Bevölkerung könnte zusätzliche verkehrsbezogene Belastungen im Haushaltsbudget nicht auffangen und wäre daher ohne Kompensationsmaßnahmen zu deutlichen Mobilitätseinschränkungen gezwungen.

\section{... und im Güterverkehr}

Die Wirkung der in den Szenarien untersuchten Maßnahmen im Güterverkehr wird dadurch relativiert, dass im Jahre 1994 die festen Kosten insgesamt (mit Fahrzeugabschreibung) im Straßengüterverkehr mehr als zwei Drittel der Gesamtkosten betrugen. Im Güterfernverkehr zeigt eine Beispielrechnung für einen typischen LkwZug (40 t) mit einem Kaufpreis von $240000 \mathrm{DM}$, dass sich bei einer durchschnittlichen jährlichen Fahrleistung von 120000 km mit hohem Autobahnanteil Road Pricing wesentlich stärker auswirkt als die jetzige Vignette. Die Mehrbelastung entspricht in der Größenordnung der in Szenario 3 unterstellten zusätzlichen Mineralölsteuererhöhung. Langfristig steigen die Lkw-Betriebskosten im Fernverkehr insgesamt durch die in den Szenarien vorgesehenen Maßnahmen um rund die Hälfte, von rd. $280000 \mathrm{DM}$ auf 420000 bis $440000 \mathrm{DM}$. Im Güternahverkehr hat von den unterstellten Maßnahmen nur die Mineralölsteuererhöhung Bedeutung. Die Kosten steigen hierdurch langfristig um $15 \%$ (Szenarien 1 und 2), bzw. $30 \%$ (Szenario 3).

Bei den Rechnungen zur Kostenbelastung sind auch die möglichen Reaktionen der Transportunternehmen mit einbezogen. Diese umfassen

- den Einsatz von verbrauchsärmeren Lkw als heute,

- sowie die Vermeidung von Leer- und schwach ausgelasteten Fahrten. 
Im Vergleich zum Pkw sind die fahrzeugbezogenen Einsparmöglichkeiten jedoch gering, da die Nutzlast einen hohen Anteil am Fahrzeuggewicht hat und keine praktikablen technischen Verfahren zur Rückgewinnung und Speicherung der Bremsenergie erkennbar sind. Die logistischen Möglichkeiten, die durchschnittliche Auslastung der Fahrzeuge zu verbessern, dürfen ebenfalls nicht überschätzt werden; in vielen Relationen ist wegen der Unpaarigkeit der Verkehrsströme und/oder dem Einsatz von Spezialfahrzeugen keine höhere Auslastung als $50 \%$ möglich.

Tabelle 4 zeigt das zusammengefasste Ergebnis der Schätzungen zu Verkehrsverlagerung und -verminderung: Trotz der drastischen Preiserhöhung bei den Betriebskosten um $50 \%$ im Fernverkehr und bis zu $30 \%$ im Nahverkehr wird die Lkw-Fahrleistung insgesamt nur um $10 \%$ verringert. Als Resultat der Verlagerungsschätzungen nach Güterbereichen ergibt sich im Fernverkehr eine Preiselastizität von -0,4. Im Nahverkehr beträgt die Reduzierung der Fahrleistung nur 2 - $5 \%$, da auch bei den auf die Schiene verlagerten Ferntransporten vielfach noch ein Vor- und Nachlauf als LkwFahrt erforderlich ist.

Tab. 4: Entwicklung der Lkw-Fahrleistungen - Mrd. Fahrzeug-km -

\begin{tabular}{|l|c|c|c|c|}
\hline & 1994 & Szenario 1 & Szenario 2 & Szenario 3 \\
\hline Nahverkehr & 39,3 & 38,3 & 38,3 & 37,5 \\
dar. Autobahn & 29,4 & 28,6 & 28,6 & 28,0 \\
& & & & \\
Fernverkehr & 21,1 & 17,3 & 17,2 & 17,0 \\
dar. Autobahn & 18,6 & 15,2 & 15,1 & 14,9 \\
& & & & \\
Insgesamt & 60,4 & 55,6 & 55,5 & 54,5 \\
dar. Autobahn & 48,0 & 43,7 & 43,6 & 42,9 \\
\hline Quelle: Berechnungen des DIW.
\end{tabular}

Das Ergebnis der relativ geringen Verkehrswirksamkeit selbst einer Preiserhöhung, die deutlich über das in der politischen Diskussion als realisierbar Diskutierte hinausgeht, mag überraschen. $\mathrm{Zu}$ berücksichtigen ist jedoch, dass
- die Bahn durch die Aufteilung in diverse Transportunternehmen mit entsprechenden Grenzaufenthaltszeiten etc. gerade bei diesen Transportströmen dem Lkw deutlich unterlegen ist;

- der Transportpreis gegenüber anderen Transporteigenschaften vielfach von untergeordneter Bedeutung ist;

- insbesondere in den Road PricingSzenarien die Qualität des Lkw-Transportes steigt. Die Transportverlagerungen verringern die Stauanfälligkeit der auf der Straße verbleibenden Transporte. In den Road Pricing-Szenarien wird über eine unterschiedliche Bepreisung darüber hinaus eine zeitlich-räumlich verfeinerte Steuerung des Verkehrsflusses möglich.

Der letztgenannte Effekt führt dazu, dass die Verlagerungen im Szenario 1 (Road Pricing auch für Pkw) am niedrigsten ausfallen. Insgesamt sind die Unterschiede zwischen den Szenarien jedoch gering.

Für die ökonomische Folgenanalyse sind vor allem die Preiseffekte der Mineralölsteuererhöhungen und die des Road Pricing auf Autobahnen und ausgewählten Fernstraßen zu berücksichtigen. Das vorgesehene Cordon Pricing für Ballungsräume hat nur punktuell $\mathrm{Be}$ deutung, z. B. zur Förderung des verstärkten Einsatzes lärmarmer Lkw oder zur Entzerrung von Verkehrsspitzen, vorausgesetzt, die Preisgestaltung kann entsprechend differenziert werden.

Die erhöhten Abgaben wirken direkt auf die Lkw-Betriebskosten, die aber, wie oben dargestellt, nur ein Teil der gesamten Transportkosten des Straßengüterverkehrs sind. Zudem sind diese Trucking-Costs nur ein Teil der Dienstleistungen der Transportunternehmen und Speditionen, wie sie in den Volkswirtschaftlichen Gesamtrechnungen (VGR) ausgewiesen werden. Der Wert dieser in den Transportpreisen enthaltenen Nebenleistungen, von der Verpackung über Lagerhaltung, Inkasso, bis zur Regalbestückung am Bestimmungsort ist nur zu schätzen, dürfte aber bei bis zu zwei Dritteln der in der Volkswirtschaftlichen Gesamtrechnung ausgewiesenen Wertschöpfungsund Produktionsgrößen des Verkehrs liegen. 
Neben der Relativierung/Einordnung der Maßnahmen sind die möglichen Anpassungsreaktionen der Verlader zu berücksichtigen. An Verbrauchsreduzierung ist, wie bereits ausgeführt, beim Lkw im Vergleich zum Pkw nur wenig möglich, daher ist in Szenario 3 beim Lkw für deutsche Unternehmen mit höherer Belastungszunahme zu rechnen als bei den Road Pricing-Szenarien (im Gegensatz zum Pkw-Verkehr). Einzubeziehen sind vor allem Verkehrsverlagerungen auf Bahn/Schiff und Änderungen der Lieferbeziehungen/Logistikketten.

Die Einnahmen des Staates aus dem LkwVerkehr würden sich durch die SzenarioMaßnahmen auf jährlich gut 70 Mrd. DM (Szenarien 1 und 2) bzw. 60 Mrd. DM (Szenario 3) verfünffachen. Ohne Anpassungsmaßnahmen der Verkehrsakteure (überwiegend durch Transportverlagerungen auf die Bahn und den Einsatz verbrauchsärmerer Fahrzeuge) wären die Einnahmen noch um rd. $10 \mathrm{Mrd}$. DM höher. Die Einnahmen im Szenario 3 der ausschließlichen Mineralölsteuererhöhung sind niedriger als in den Road Pricing-Szenarien, u. a. da im Ausland getankt werden kann.

Der Anteil der Lkw-Transporte am gesamtwirtschaftlichen Produktionswert ist relativ niedrig; er lag 1994 bei rund $1 \%$. Wesentliche Bedeutung haben die Transportkosten nur für die Bauwirtschaft (Anteil etwa $11 \%$ ). Auch für die Land- und Forstwirtschaft sowie die Nahrungsmittel- und Getränkeindustrie haben die Transportkosten mit einem Anteil von rund $3 \%$ noch einen gewissen Stellenwert. In den meisten Sektoren jedoch haben die Transportleistungen kostenmäßig nur ein geringes $\mathrm{Ge}-$ wicht. Aus diesem Grund hat der Transportpreis nur eine minimale Wirkung auf die Höhe des gesamten Verkehrsaufkommens und auch die Verminderungswirkung im Sinne kürzerer Transportentfernungen ist gering.

Die gesamtwirtschaftliche Bedeutung der Maßnahmen lässt sich durch die Preiswirkungen auf die Komponenten der Endnachfrage kennzeichnen. Überdurchschnittlich (um $1,7 \%)$ steigen hier die Preise der Exporte. Neben den im Warenwert indirekt enthaltenen Transportleistungen sind sie auch durch die direkten Verkehrsleistungen belastet. Der private Verbrauch würde sich, zusätzlich zu den auf den Pkw wirkenden Maßnahmen der Szenarien, durch die Lkw-Transportkostensteigerungen um $0,7 \%$ verteuern (vgl. Tab. 5).

Tab. 5: Wirkung der Lkw-Transportkostenerhöhung - Insgesamt

\begin{tabular}{|l|l|l|l|}
\hline & \multicolumn{3}{|c|}{ Preiseffekt $^{1)}$ in \% } \\
& Szenario 1 & Szenario 2 & Szenario 3 \\
\hline Privater Verbrauch & 0,70 & 0,70 & 0,69 \\
Staatsverbrauch & 0,38 & 0,38 & 0,38 \\
Ausrüstungsinvesti- & 0,84 & 0,84 & 0,83 \\
tionen & 0,90 & 0,90 & 0,90 \\
Bauteninvestitionen & 1,67 & 1,67 & 1,65 \\
Ausfuhr & 0,88 & 0,88 & 0,87 \\
Insgesamt & \multicolumn{3}{|l|}{} \\
\hline I) Verteuerung bei vollständiger Preisüberwälzung. \\
Quellen: Statistisches Bundesamt; Berechnungen des DIW. \\
\hline
\end{tabular}

Bei den sektoralen Preiseffekten sind die Unterschiede zwischen den Szenarien so gering, dass eine getrennte Darstellung nicht sinnvoll ist. Bei völliger Überwälzung der nach Anpassungsreaktionen verbleibenden Mehrbelastung ergeben sich die größten Preiserhöhungen mit $3,1 \%$ bei Baustoffen. Hier steigt der LkwAnteil am Produktwert unter Einbeziehung der indirekten Kostenbestandteile auf $14 \%$. Der Nahverkehrsanteil ist bei diesen Transporten zwar hoch (und damit der Autobahnanteil niedrig), aber es bestehen im Baustellenverkehr über relativ kurze Strecken nur geringe Verlagerungspotenziale vom Lkw auf andere Verkehrsarten. Die Ergebnisse zeigen, dass die Endpreise in fast allen übrigen Bereichen nur geringfügig steigen. Diesen Mehrbelastungen stehen die entsprechenden staatlichen Mehreinnahmen gegenüber und eine Entlastung des Straßennetzes um jeden zehnten Lkw. 
Zusammengefasst zeigen die Ergebnisse, dass die Wirkungen auf die Verkehrsnachfrage im Güterverkehr in den drei Szenarien nahezu gleich sind (vgl. Tab. 6). Ebenso wie Road Pricing ist die Mineralölsteuer entfernungsabhängig, bietet also den Anreiz, lange Transporte auf andere Verkehrsträger als den Lkw zu verlagern. Die Szenarien 1 und 2 der gemischten Strategie von Road Pricing und Mineralölsteuer unterscheiden sich im Güterverkehr ohnehin nur dadurch, dass in Szenario 1 der Pkw-Verkehr etwas stärker reduziert wird, so dass die Bedingungen für einen reibungslosen Lkw-Transport geringfügig günstiger sind. Auch in den ökonomischen Wirkungen stimmen die drei Szenarien damit weitgehend überein.

Tab. 6: Zusammenfassung der Ergebnisse zum Güterverkehr im Jahre 1994 in Deutschland und in den Szenarien

\begin{tabular}{|c|c|c|c|c|c|}
\hline & Einheit & 1994 & Szenario 1 & Szenario 2 & Szenario 3 \\
\hline \multicolumn{6}{|l|}{ Verkehrsausgaben 1) } \\
\hline Bahn / Binnenschiff & Mrd. DM & 11,0 & 14,9 & 14,9 & 15,2 \\
\hline Lkw & Mrd. DM & 93,0 & 144,0 & 144,0 & 133,0 \\
\hline Verkehr insgesamt & Mrd. DM & 104,0 & 158,9 & 158,9 & 148,2 \\
\hline \multicolumn{6}{|l|}{ Verkehrsleistungen } \\
\hline Bahn / Binnenschiff & Mrd. tkm & 132,9 & 160,1 & 160,1 & 162,5 \\
\hline Lkw & Mrd. tkm & 229,1 & 200,9 & 200,9 & 198,5 \\
\hline Verkehr insgesamt & Mrd. tkm & 362,0 & 361,0 & 361,0 & 361,0 \\
\hline \multicolumn{6}{|l|}{ Fahrleistungen } \\
\hline Lkw & Mrd. km & 60,4 & 55,6 & 55,5 & 54,5 \\
\hline dav.: Bundesautobahnen & Mrd. km & 48,0 & 43,7 & 43,6 & 42,9 \\
\hline Übrige Straßen & Mrd. km & 12,4 & 11,9 & 11,9 & 11,6 \\
\hline \multicolumn{6}{|l|}{ Kraftstoffverbrauch } \\
\hline Lkw & Mrd. 1 & 18,0 & 14,9 & 14,9 & 13,7 \\
\hline \multirow{2}{*}{\multicolumn{5}{|c|}{ Verkehrsausgaben }} & \\
\hline & & & & & \\
\hline Bann / BinnenscnirI & $\%$ & $\mathrm{X}$ & 35,8 & 35,8 & 38,8 \\
\hline Lkw & $\%$ & $\mathrm{X}$ & 54,8 & 54,8 & 43,0 \\
\hline Verkehr insgesamt & $\%$ & $\mathrm{x}$ & 52,8 & 52,8 & 42,6 \\
\hline \multicolumn{6}{|l|}{ Verkehrsleistungen } \\
\hline Bahn / Binnenschiff & $\%$ & $\mathrm{x}$ & 20,5 & 20,5 & 22,3 \\
\hline Lkw & $\%$ & $\mathrm{x}$ & $-12,3$ & $-12,3$ & $-13,4$ \\
\hline Verkehr insgesamt & $\%$ & $\mathrm{X}$ & $-0,3$ & $-0,3$ & $-0,3$ \\
\hline \multicolumn{6}{|l|}{ Fahrleistungen } \\
\hline Lkw & $\%$ & $\mathrm{x}$ & $-7,9$ & $-8,1$ & $-9,8$ \\
\hline dav.: Bundesautobahnen & $\%$ & $\mathrm{x}$ & $-9,0$ & $-9,2$ & $-10,6$ \\
\hline Übrige Straßen & $\%$ & $\mathrm{X}$ & $-4,0$ & $-4,0$ & $-6,5$ \\
\hline \multicolumn{6}{|l|}{ Kraftstoffverbrauch } \\
\hline Lkw & $\%$ & $\mathrm{X}$ & $-17,2$ & $-17,2$ & $-23,9$ \\
\hline
\end{tabular}


Wie in bisherigen Untersuchungen zum Güterverkehr wird deutlich, dass der Preis nur eine minimale Wirkung auf die Höhe des gesamten Verkehrsaufkommens hat und auch die Verminderungswirkung im Sinne kürzerer Transportentfernungen sehr gering ist. Von großer Bedeutung sind die Preise dagegen für die Anteile der Verkehrsarten. Für Verkehrsverlagerungen sind die Veränderungen der Preisrelationen zwischen den konkurrierenden Verkehrsarten in dem hier zugrunde gelegten Ausmaß neben den genannten weiteren Anforderungen an den Transport mit entscheidend.

Road Pricing bietet erweiterte Möglichkeiten des Verkehrsmanagements, insbesondere wenn es mit Hilfe moderner IuK-Techniken implementiert wird. Darüber hinaus werden ausländische Lkw stärker in die Abgabenerhebung einbezogen, als bei der Mineralölsteuer. Road Pricing hat einen klaren Ortsbezug, wie die historische Erhebung von Wegezöllen. Damit bietet sich ggf. eine ortsbezogene Kompensation für die mit dem Durchgangsverkehr zu ertragenden Belastungen an. Ein Hauptproblem des Fernverkehrs liegt ja darin, dass die an den Verkehrswegen wohnenden Personen den Flächenverbrauch, Lärm und die anderen Emissionen ertragen müssen, ohne einen direkten Nutzen zu haben.

Für den Verzicht auf die Einführung von Autobahngebühren sprechen die entfallenden Investitions- und Betriebskosten, sowie der von drastischen Mineralölsteuererhöhungen verstärkt ausgehende Anreiz zu Entwicklung und Einsatz verbrauchsärmerer Fahrzeuge. Bei gleicher Preiselastizität in den Szenarien ergibt sich eine etwas höhere Verlagerungswirkung als beim Road Pricing, da die hier erwarteten Attraktivitätsverbesserungen im Straßenverkehr entfallen.

Ein wesentliches Argument für eine parallele Erhebung von Mineralölsteuer und Einführung von Autobahngebühren ist die damit entstehende Transparenz:
- Road Pricing ist die Abgabe zur Benutzung der Infrastruktur und zur Engpasssteuerung. Die Abnutzung der Straßen ist überwiegend auf die Lkw und nicht die Pkw zurückzuführen, so dass auch die alleinige Realisierung für Lkw, wie zurzeit politisch vorgesehen und hier in Szenario 2 betrachtet, vertretbar erscheint.

- Die Mineralölsteuer ist, wie andere Verbrauchssteuern auch, neben einer Einnahmequelle ein Lenkungsinstrument, um den Verbrauch von Gütern zu belasten. Die bestehende teilweise Zweckbindung kann schrittweise mit der Einführung von Road Pricing aufgehoben werden.

Dies spricht für eine Umsetzung eines Road Pricing-Szenarios mit Straßenbenutzungsgebühren entsprechend der Höhe der Wegekosten, flankiert durch eine stetige Anhebung der Mineralölsteuer als allgemeine, nicht zweckgebundene Steuer, um Anreize für einen verantwortungsvollen Umgang mit nicht erneuerbaren Rohstoffen zu geben.

\section{Anmerkung}

1) Kuhfeld, Hartmut; Schlör, Holger; Voigt, Ulrich: Ökonomische Folgenanalyse im Rahmen des TAB-Projektes „Optionen zur Entlastung des Verkehrsnetzes und zur Verlagerung von Straßenverkehr auf umweltfreundlichere Verkehrsträger". Gutachten im Auftrage des Büros für Technikfolgen-Abschätzung beim Deutschen Bundestag (TAB). Berlin 1996 (als Manuskript vervielfältigt).

\section{Kontakt}

Dr. Ulrich Voigt

Deutsches Institut für Wirtschaftsforschung

Abteilung Verkehr

Königin-Luise-Straße 5, D-14191 Berlin (Dahlem)

Tel.: + 49 (0) $30 / 89789$ - 311

Fax: + 49 (0) $30 / 89789$ - 103

E-Mail: u voigt@diw.de 